January 2, 2003

\title{
NASA Thermal Control Technologies for Robotic Spacecraft
}

\section{Theodore D. Swanson* and Gajanana C. Birur **}

* National Aeronautics and Space Administration, Goddard Space Flight Center, Code 540, Greenbelt, MD 20771, USA

Phone: 301-286-7854, Fax: 301-286-1717, E-mail: Theodore.D.Swanson@nasa.gov

**Jet Propulsion Laboratory, California Institute of Technology, 4800 Oak Grove Drive, Pasadena CA 91109, USA

Phone: 818-354-4762, E-mail: Gajanana.C.Birur@jpl.nasa.gov

\begin{abstract}
Technology development is inevitably a dynamic process in search of an elusive goal. It is never truly clear whether the need for a particular technology drives its development, or the existence of a new capability initiates new applications. Technology development for the thermal control of spacecraft presents an excellent example of this situation. Nevertheless, it is imperative to have a basic plan to help guide and focus such an effort. Although this plan will be a living document that changes with time to reflect technological developments, perceived needs, perceived opportunities, and the everchanging funding environment, it is still a very useful tool. This presentation summarizes the current efforts at NASAVGoddard and NASAJPL to develop new thermal control technology for future robotic NASA missions.
\end{abstract}

Key Words: advanced thermal control, capillary pumped loops, loop heat pipes, variable emissivity surface, cryogenic, heat switches, thermal storage 


\section{INTRODUCTION}

The National Aeronautics and Space Administration's (NASA) Goddard Space Flight Center and Jet Propulsion Laboratory primarily support the agency's Space Science Enterprise and Earth Science Enterprise missions. These are robotic, unmanned missions that typically are in Earth orbit, at a Lagrangian point, or travel to another planet. All these missions have one overriding purpose: the pursuit of new scientific knowledge. NASA is continually trying to look further out into space and time, into new parts of the spectrum, investigate alien planetary and solar environments, gain better resolution than ever before, and pursue scientific objectives. Clearly, the pursuit of such goals requires advanced engineering to make it all possible. This is particularly true for thermal control. Many of the proposed future missions involve science that can only be obtained at deep cryogenic temperatures, with extreme dimensional stability of the spacecraft and instrument, typically with very tight and stable temperature control, and with related requirements that drive the need for new thermal control technology. Such technology is also needed to minimize mass and power and create more reliable and robust space science missions to explore deep space and other planets,

\section{STATE-OF-THE-ART IN THERMAL CONTROL}

Two-phase technology, such as Capillary Pumped Loops (CPLS) and Loop Heat Pipes (LHPs), is clearly the major thermal control innovation of the last decade and as such defines the state-of-the-art ${ }^{1,2,3}$. This technology has now gained acceptance and is used in a variety of NASA and commercial applications. For example, NASA's TERRA 
spacecraft, which was launched in December of 1999, has three CPLs that have been in successful operation since launch ${ }^{4}$. In July of 2001 one of the instruments that is thermally conditioned by a CPL, the ASTER-SWIR instrument, began to experience excessive temperatures. The temperature control point of its CPL was adjusted in-orbit to accommodate this temperature growth, thus extending the instrument's life. Other recent CPL applications include the Hubble Space Telescope, which has a CPL to remove heat from the new NICMOS cryocooler that is located inside the aft shroud ${ }^{5}$. On a recent shuttle mission, HST/Servicing Mission-3B in March of 2002, the astronauts installed a CPL evaporator through the bottom of the aft shroud, attached it to the new NICMOS cryo-cooler, and then attached a new radiator to the external handrails. This system is depicted in Figure 1. Flight data has verified that the new cryo-cooler and CPL cooling system has successfully reduced the temperature of the NICMOS sensors to approximately $70 \mathrm{~K}$, which is the temperature needed for operation ${ }^{6}$.

Current LHP applications include the TES instrument on the AURA spacecraft, the GLAS instrument on the ICESAT spacecraft, and the BAT instrument on the SWIFT instrument. The GLAS instrument is a laser operating at room temperature with approximately 120 watts thermal load, and is scheduled for launch in early $2003^{7}$. The GLAS LHP application is depicted in Figure 2. In the commercial sector Boeing Space Systems has used LHPs with deployable radiators on its series 702 communication spacecraft.

All current CPL and LHP applications involve the use of a single evaporator. This approach is somewhat limiting. To address this issue, the CAPL 3 Flight Experiment was flown on the Shuttle in December of $2001^{8}$. The CAPL 3 experiment successfully demonstrated the use of parallel evaporators with heat load sharing between them. It 
had four, $2.5 \mathrm{~cm}$ diameter evaporators with polyethylene wicks, a separate starter pump, eight direct condenser lines plumbed in parallel, and a back pressure flow regulator to facilitate clearing of the evaporators during startup. One of the evaporators is connected to a cold sink via a variable conductance heat pipe and can thus be operated in a condenser mode. While in orbit CAPL 3 demonstrated reliable start-up ( 9 attempts, all successful), continuous operation for extended periods of time, high power to $1500 \mathrm{~W}$, extended low power at $100 \mathrm{~W}$, and heat load sharing between the evaporators. Overall the test was quite successful. Prior to flight the experiment was held in storage, in a fully charged state for 2 years and showed no evidence of non-condensable gas generation effects. A view of CAPL 3 from the International Space Station is shown in Figure 3. A sample of the flight data for a high power test is presented in Figure 4.

As these flight applications and experimentation with advanced system concepts demonstrates, CPLs and LHPs have reached a point of flight maturity. This is not to suggest that additional technology development is not needed, or that no implementation issues remain. For example, current issues with CPL/LHP technology include the need for a significant amount of custom engineering to design and integrate the technology, and preconditioning of the loop is often required prior to start-up. Irrespective of these concerns, current two-phase technologies can be reliably applied and offer significant design flexibility, tight temperature control, broad heat transport capacity, diode function and isothermalization. Their performance is unmatched by any other technology. CPLs and LHPs solve design problems!

Another thermal technology that is providing a robust solution for short-term missions of one to two years is the mechanically pumped, single-phase cooling system. In this approach a mechanical pump circulates a single-phase liquid to remove heat from the 
hot engineering and science equipment, and then reject it at an external radiator. This system has been used in Space Shuttle for flight durations of two to three weeks. It was first used in a long-term robotic mission, the Mars Pathfinder, in 1996. See Figure 5. In this application the single-phase pumped cooling system provided a robust thermal design for the operation of the spacecraft and made the integration and testing much simpler. The pump supported the spacecraft's thermal needs and operated continuously for over seven months during the cruise to Mars. The same pumped cooling system will be used on the Mars Exploration Rover mission, which will send two rovers on separate spacecraft to Mars in 2003.

\section{FUTURE THERMAL CONTROL REQUIREMENTS}

While advanced two-phase technology such as CPLs and LHPs offer major advantages over traditional thermal control technology, it is clear that this technology alone will not meet the needs of all future scientific spacecraft. Emerging trends in spacecraft and instrument design are thus engendering very challenging thermal control requirements, which require increasingly sophisticated thermal control technology. Major drivers include:

- Dimensional stability of large structures

- Deep cryogenic heat acquisition and transport (40 $\mathrm{K}$ and below)

- Tight temperature control $\left(+/-1^{\circ} \mathrm{C}\right)$

- Integrated thermal/mechanical/optical systems

- Common thermal design for fleets of micro/nano spacecraft

- High flux heat acquisition $\left(>100 \mathrm{~W} / \mathrm{cm}^{2}\right)$ with tight temperature control

- Challenging thermal sink situations, especially for planetary environments 
- Minimization of mass and auxiliary power use

- Thermal control of inflatable and gossamer space structures

- Thermal control of spacecraft in extreme high temperature environments

Based on surveys of proposed future robotic missions, it is clear that cryogenic structures and other large-scale applications (down to a few Kelvin) are an emerging trend, while stringent optical alignment and sensor needs are requiring ever-tighter temperature control. Heat flux levels from lasers and other similar devices are increasing. Additionally, planetary environments and missions close to the sun will create unique thermal challenges that will require very innovative thermal control technologies.

Large distributed instruments such as mirrors will also require also creative techniques to integrate structural, mechanical alignment, and thermal control functions. In order to keep the signal to noise ratio sufficiently low when viewing objects in the near to far infrared and sub-millimeter region of the spectra, the entire optical path must be kept very cold. For example, much of the optics for the James Webb Space Telescope must be in the $50 \mathrm{~K}$ or below range ${ }^{9}$. Conceptual designs for even more advanced concepts call for optical paths as cold as a few degrees Kelvin. Providing cooling of large optics to such levels will truly be a very challenging task. Figure 6 depicts one concept of an integrated thermal control system that includes a sunshield, high conductivity substrate, cryogenic heat pipes, cryogenic LHPs, and active cryo-coolers.

Nano and micro spacecraft will also drive the need for new technologies, particularly since such small spacecraft will have low thermal capacitance. This situation, combined with the need for tighter temperature control, will present a challenging design problem 
when such spacecraft/instruments undergo thermal transients. The ST-5 spacecraft, scheduled to be launched by NASA in 2004, includes a demonstration of innovative thermal control technologies for such small ( $<25 \mathrm{~kg}$ class) spacecraft.

The use of "off-the-shelf" commercial spacecraft buses for demanding science instruments may also presents various challenges. Most commercial spacecraft busses are not designed for the stability and temperature control needs of sophisticated science instruments.

\section{EMERGING TECHNOLOGIES}

In response to these perceived needs NASAGoddard and NASAJJPL are pursuing a variety of thermal control technology development efforts to identify and mature, through flight demonstration as appropriate, promising new thermal control technologies for robotic spacecraft. Many of these technologies could also be adapted for use on manned missions. Current efforts include:

- Advanced thermal control coatings such as variable emissive surfaces that permit adaptive, intelligent control of a radiator's capacity.

- Cryogenic ( $3 \mathrm{~K}$ to $80 \mathrm{~K}$ ) heat transport devices (loop heat pipes, capillary pumped loops, etc.) for sensor and /or optics cooling which incorporate a diode function.

- Integrated structural, alignment, and thermal control concepts for very large structures.

- Thermal switches for planetary and/or cryogenic applications 
- Advanced high conductivity materials, such as diamond films, which may be suitable for cryogenic applications.

- Multi-evaporator/multi-condenser, two-phase heat transport loops

- Techniques, such as spray cooling, capable of heat removal rates $>100 \mathrm{~W} / \mathrm{cm}^{2}$

- Long-life mechanical pumps for single and two-phase pumped cooling loops

- Lightweight, high performance thermal insulation material for planetary environments

- MEMS-based pumped cooling systems for high power density heat removal

- Phase change thermal storage material for low and high temperature applications

Special thermal control coatings that can change their effective emissivity in response to a controlled signal may be the next major innovation in thermal control. System studies have demonstrated that this technology is capable of savings in make-up heater power in excess of $90 \%$, and/or weight savings of over $75 \%{ }^{10}$. These technologies are generically applicable to all spacecraft but are especially suitable for micro/nano spacecraft with very limited power and mass allocations. At least three different technologies are currently under development; electrochromic, electrostatic, and a micro-louver. All three technologies are planned to be flown on NASA's ST-5 spacecraft in $2004^{11}$. The electrochromic approach involves the use of a conducting polymer on a thin film with a small bias voltage used to change the redox state. The electrostatic concept involves a thin film that has an electrically conductive coating on the inside and a white paint on the outside. It can be electrostatically held off a radiative surface to act as a single layer of multi-layer insulation, or it can be held tight against the surface and radiate heat efficiently. The micro-louver is functionally very similarly to a traditional louver, except that the louvers are on the scale of microns. It is based on 
microelectromechanical (MEMS) technology. The goal with all three concepts is to demonstrate variable emittance technologies that have a net change in effective emissivity of 0.4 or more and can survive the space environment. As survival in space is expected to be a rather challenging requirement, multiple technologies are being pursued to reduce programmatic risk. All three technologies are currently making good progress towards these goals.

Cryogenic CPLs and LHPs offer numerous design options. These devices would allow the sensor to be separated from the cryo-cooler, thus reducing induced vibration and electromagnetic interference from the cryo-cooler. Since the cryogenic sensor(s) could now be connected to a group of cryo-coolers, redundancy could be improved while reducing the total number of cryo-coolers used. Improved packaging is also possible because the sensor can now more easily be located in the heart of the instrument while the heavy, and thermally hot, cryo-coolers can be located on the skin (or on the sun side of a sunshield). A cryogenic CPL/LHP would also allow a diode function to prevent back heat leaks if the cryo-cooler were turned off or malfunctioned.

A cryogenic CPL was flown on the Shuttle flight STS-95 in $1998^{12}$. It successfully demonstrated reliable start-up, and heat transport of over 2.5 watts at approximately 80K. See Figure 7. Transport on the ground was over 10 watts; the flight experiment was limited due to the available cold sink. The test unit used nitrogen as a working fluid, weighed 191 grams, and had an effective transport length of 0.25 meters.

Other cryogenic loops with neon and hydrogen have been built and ground tested with good results. Neon CPLs operating at approximately 30 to $38 \mathrm{~K}$ have demonstrated 1 to 5 watts of transport, while hydrogen based systems operating at approximately 18 to 25 
$K$ have demonstrated transport capacities in excess of 1 watt. Diode action has also been demonstrated. The long term goal is to develop a helium based, cryogenic twophase loop that can transport a few milliwatts at 2 to $4 \mathrm{~K}$.

Thermal switches, either for isolation of cryogenic devices in a warm environment or to keep warn objects from getting too cold in a cold environment, are also under development. While different approaches to this device have been attempted over the years, current research is focusing on wax-actuated devices that make/break a contact in response to an external signal. Miniature heat switches for nanosat and Mars rover applications are a key technology for such applications ${ }^{13,14}$. Target performance is for a conductance of $0.4 \mathrm{~W} / \mathrm{C}$, a switching ratio of 30 in an 8 torr $\mathrm{CO} 2$ atmosphere, and a weight of less than 120 grams. Figure 8 depicts a current design concept for such a thermal switch.

Materials with ultra high thermal conductivity are of interest primarily for use as heat spreaders. The carbon vapor deposited (CVD) form of diamond is one material that offers considerable promise. Diamond is the hardest known material, it has the highest thermal conductivity of any material (about 4 times that of copper and about 20 times copper's thermal diffusivity), has excellent mechanical strength, is a superb electrical isolator, and also has the lowest coefficient of friction of any known material. CVD diamond is very close to natural diamond in many of these properties and is becoming less expensive and easier to apply to more materials. Metal matrix composites using very low cost diamond dust or carbon nanotubes as a filler are another possibility. These composites have thermal and mechanical properties that are superior to aluminum. Clearly there is a wide range of space applications for such materials, such as the first stage of a heat collection/isothermalization system for large aperture 
telescope mirrors or as substrates for electronic cards. For example, CVD diamond was recently evaluated, with good results, as a diode heat spreader for an application on the Hubble Space Telescope (HST).

While current CPL and LHP applications have been limited to loops with a single evaporator, clearly there are many applications where a multi-evaporator loop would offer significant benefits. The CAPL 3 flight experiment discussed earlier, which had 4 large diameter evaporators in parallel, successfully demonstrated this concept. Some future mission concepts, such as those that involve long booms that must be geometrically stable regardless of thermal loading, may require two-phase loops with many more evaporators in parallel than this experiment.

A variety of applications, such as lasers, electronic chips, and advanced propulsion devices, are expected to involve high heat fluxes (above $100 \mathrm{~W} / \mathrm{cm}^{2}$ ). These applications may require new heat acquisition devices, or at least modifications to current two-phase technology. Spray cooling technologies and electrohydrodynamic (EHD) pumping concepts are currently being investigated to meet these requirements. While spray cooling is currently being developed for ground applications, the issue of spray management in a zero gravity environment is a significant issue for space applications that has yet to be addressed.

Recent technology development efforts in EHD have resulted in greatly improved pumping capabilities (to several kilo Pascal). Also, a liquid nitrogen-based loop has been developed and successfully demonstrated. EHD pumped cooling systems have the advantage of very low power consumption, simple control, and low acoustic noise. 
However, unless they are very small devices EHD pumps require high voltages (in the thousands of volts).

MEMS based pumped cooling systems are also being investigated for removing heat from high power density electronics and science packages. Heat sinks with microchannels etched in silicon are attached directly to the heat sources and remove heat by circulating a liquid through the micro-channels.

The successful flight of the single phase mechanically pumped cooling system on the Mars Pathfinder spacecraft and the benefits offered by mechanically pumped cooling systems has encouraged thermal control engineers to explore this option for long duration space missions. Long-life, mechanical pumps suitable for space missions are being developed. In one technology, a pump with no bearings and seals is being tested for its suitability to future long-duration planetary missions.

In the area of phase change material (PCM) storage, two technologies based on paraffin waxes are being investigated. Dodecane (melting point, $-10.5 \mathrm{C}$ ) and hexadecane (melting point $18.5 \mathrm{C}$ ) are used in thermal storage capsules for controlling the battery temperatures in the future Mars rovers. Such solid-to-liquid PCMs offer valuable options for some thermal control problems. PCMs utilize the latent heat of melting/solidification to dampen out temperature excursions that are cyclical in nature. Thus, temperature extremes of modest duration can be avoided. The benefits are specific to the exact application. Representative hardware for a proposed battery application is depicted in Figure 9. 
One of the key elements to PCM storage technology is the high conductive matrix inside the PCM storage unit that can provide a good conductive path and also survive several hundreds of diurnal thermal cycles expected on the Martian surface operation for the future rovers.

\section{CONCLUSION}

Two-phase, ambient temperature, single/multiple evaporator thermal control loops are here and are gaining increasing acceptance for flight applications. Numerous examples of single evaporator applications are already in-orbit and several others are in assembly. This technology is proving itself to be highly versatile and offers a performance capability unmatched by conventional thermal control technology. In response to the perceived requirements of future missions, a variety of new thermal control technologies are being developed. Chief among these are the variable emittance thermal control coatings, which probably represent the next major advance in thermal control. Other technologies actively being developed include cryogenic two-phase loops (down to $3 \mathrm{~K}$ ), thermal switches, ultra high thermal conductivity materials, mechanically pumped two-phase cooling systems, phase change material thermal storage, and technologies to accommodate very high heat fluxes. Rich opportunities exist as these and other technologies offer increasing options for the thermal engineer.

\section{Acknowledgments}

A part of the research described in this paper was performed at the Jet Propulsion Laboratory, California Institute of Technology, Pasadena, under a contract with the National Aeronautics and Space Administration. 


\section{REFERENCES}

1. Ku, J., "Operating Characteristics of Loop Heat Pipes," SAE Paper No. 1999-01-2007.

2. Swanson, T., "NASAGGoddard Thermal Control Technology Roadmap-2002";

$13^{\text {th }}$ Annual Spacecraft Thermal Control Workshop, Aerospace Corporation, El Segundo, CA; March, 2002

3. Birur, G., "JPL Advanced Thermal Control Technology Roadmap"; $13^{\text {th }}$ Annual Spacecraft Thermal Control Workshop, Aerospace Corporation, El Segundo, CA; March, 2002

4. Chalmers, D., Fredley, J. and M. Kurtz, "A New Era of Instrument Thermal Management with Capillary Heat Transport Systems," $51^{\text {st }}$ International Astronautical Congress, October 2-6, 2000, Rio de Janeiro, Brazil.

5. McIntosh, R., Kaylor, M., Buchko, M., Kroliczek, E., and R. Smith, “A Capillary Pump Loop Cooling System for the NICMOS Instrument," SAE Paper No. 981814, 1998.

6. Buchko, M., and M. Kaylor, "Orbital Operational Status of the HST NICMOS CPL," 2002 International Two-Phase Thermal Control Technology Workshop, September 2426, 2002, Mitchellville, Maryland. 
7. Baker, C., Butler, D., Ku, J., and E. Grob, "Acceptance Thermal Vacuum Tests of the GLAS Flight Loop Heat Pipe Systems," STAIF 2001, American Institute of Physics, Albuquerque, New Mexico, February 11-14, 2001.

8. Ottenstein, L., Butler, D., and J. Ku, "Flight Testing of the Capillary Pumped Loop 3 Experiment," 2002 International Two-Phase Thermal Control Technology Workshop, September 24-26, 2002, Mitchellville, Maryland.

9. Parrish, K., and S. Thomson, "Cryogenic Thermal Design Overview of the 30K Passively Cooled Integrated Science Module (ISIM) for NASA's Next Generation Space Telescope", SPIE 47 ${ }^{\text {th }}$ Annual Meeting, Cryogenic Optical Systems and Instrumentation Conference, July 2002, Seattle, Washington.

10. Grob, L.M. and T.D. Swanson, "Parametric Study of Variable Emissivity Radiator Surfaces", STAIF-2000 Proceedings, 2000.

11. Douglas, D. M., Swanson, T., Osiander, R., Champion, J., Darrin, A., Biter, W, and P. Chandrasekhar, "Development of the Variable Emittance Thermal Suite for the Space Technology 5 Microsatellite"; Space Technology and Applications International Forum, Albuquerque, NM; Feb 2002

12. Bugby, D., Kroliczek, E., Ku, J., Swanson, T. D., Lt. Tomlinson, B. J., Davis, T. M., Baumann, J., and B. Cullimore, “Design and Testing of the Cryogenic Capillary Pumped Loop Flight Experiment”; Space Technology and Applications International Forum, Albuquerque, NM; Feb 1999 
13. Birur, G., Novak, K., Pauken, M., and E. Sunada; "Wax Actuated Heat Switch Development"; $13^{\text {th }}$ Annual Spacecraft Thermal Control Workshop, Aerospace Corporation, El Segundo, CA; March, 2002

14. Lankford, K., and E. Sunada, "Paraffin Actuated Heat Switch Flight Qualification"; $13^{\text {th }}$ Annual Spacecraft Thermal Control Workshop, Aerospace Corporation, El Segundo, CA; March, 2000. 


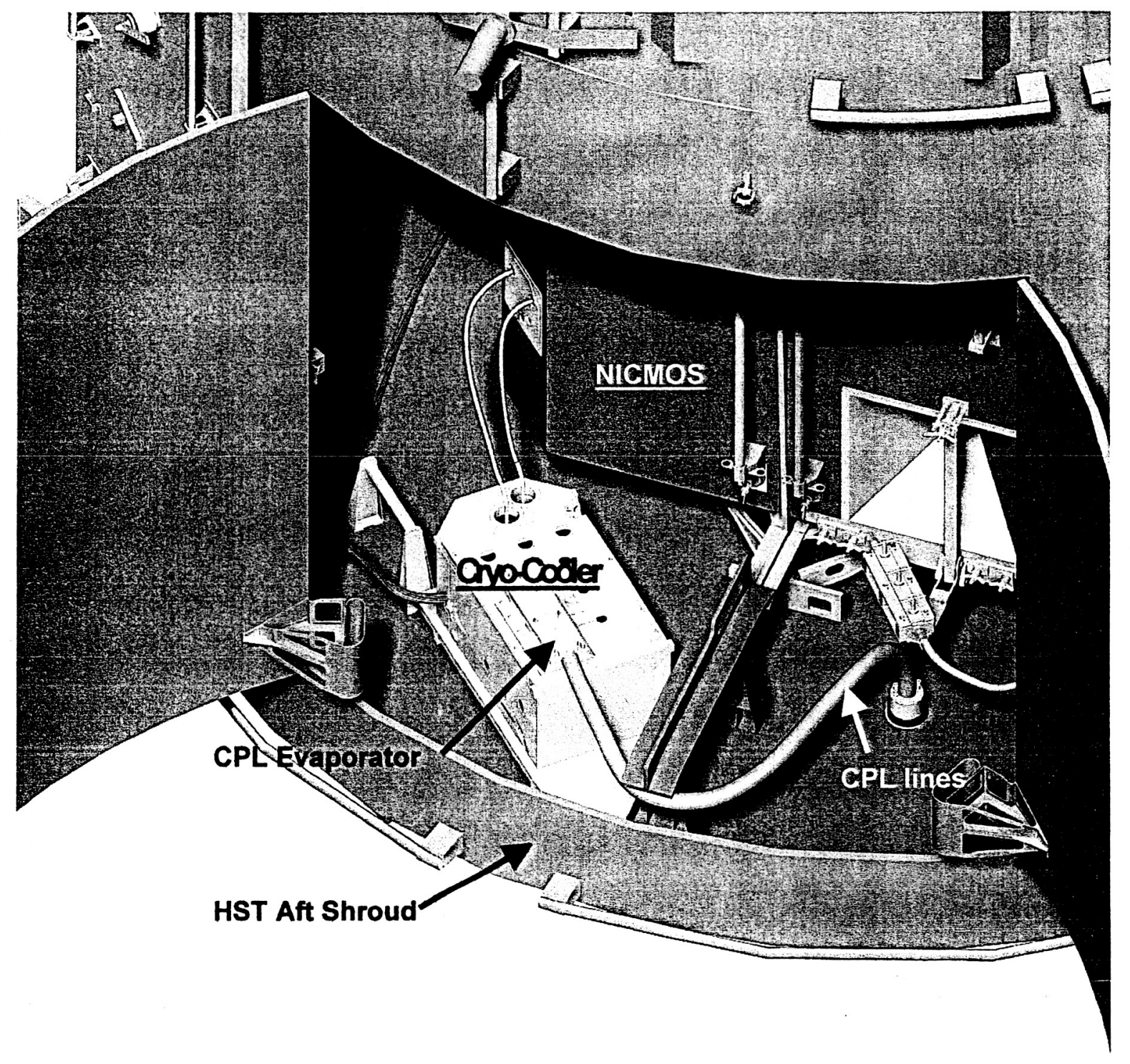

Figure 1: CPL and cryo-cooler on the Hubble Space Telescope 


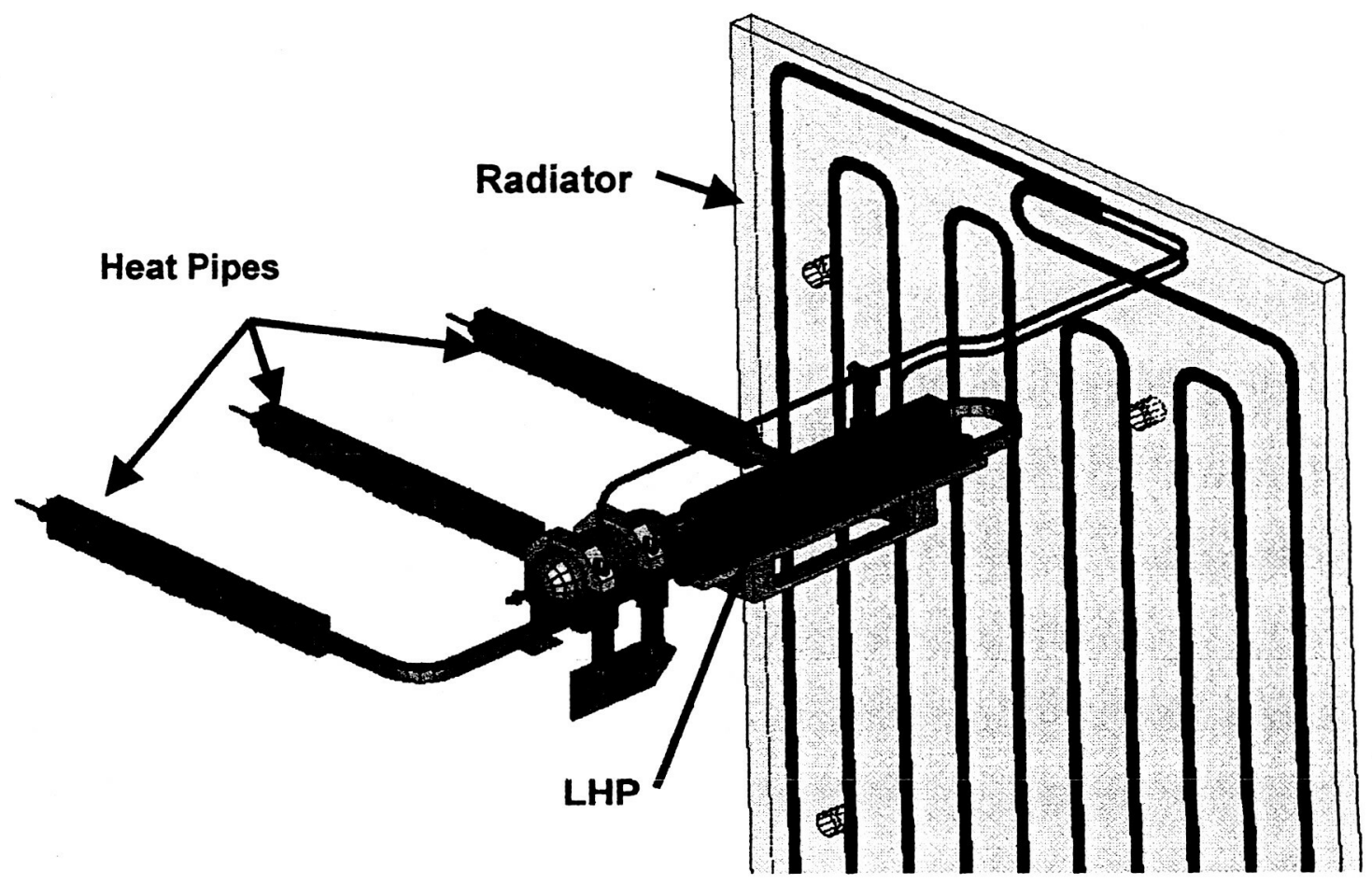

Figure 2: LHP Application on NASA's GLAS laser instrument on ICESAT 


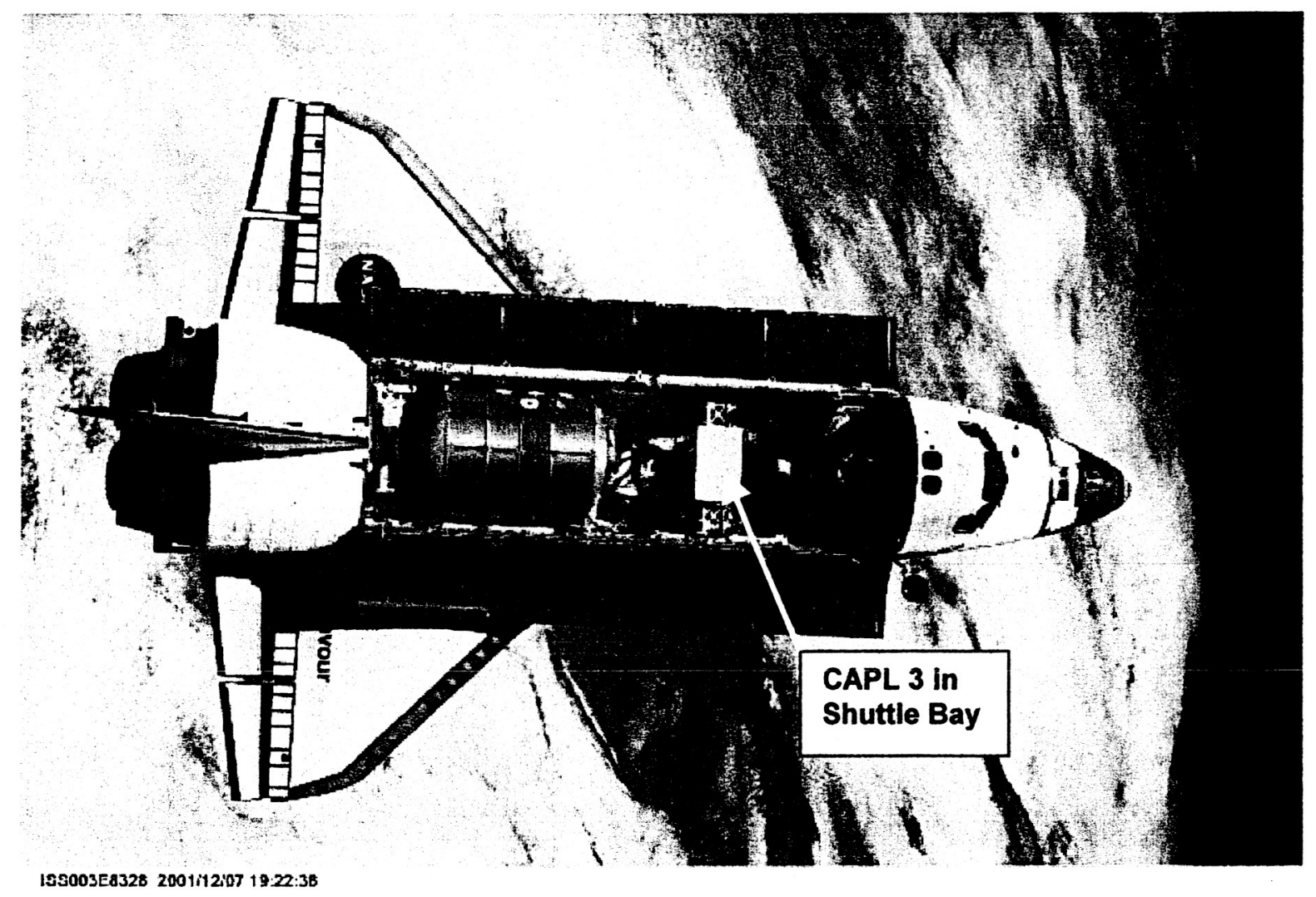

Figure 3: CAPL 3 in Shuttle Bay on STS-108 
HIgh Power 12/16/201

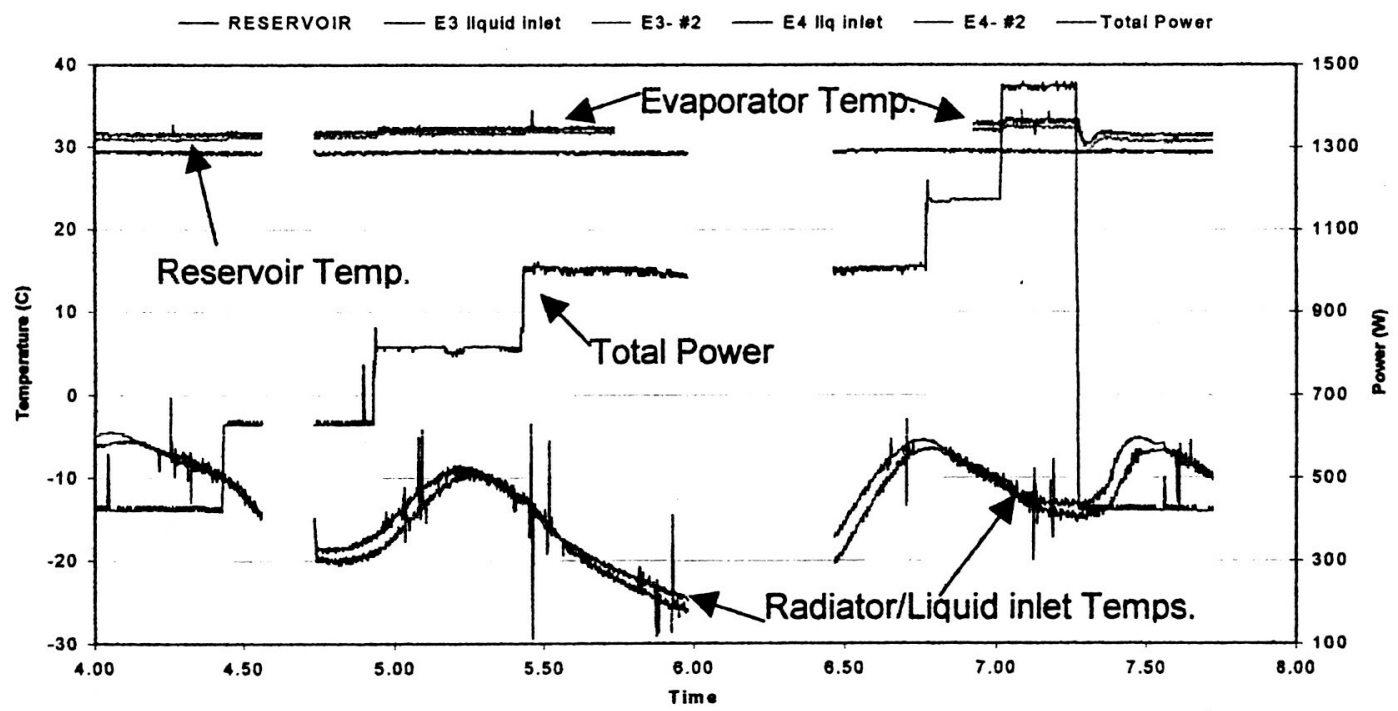

Figure 4: High Power test of CAPL 


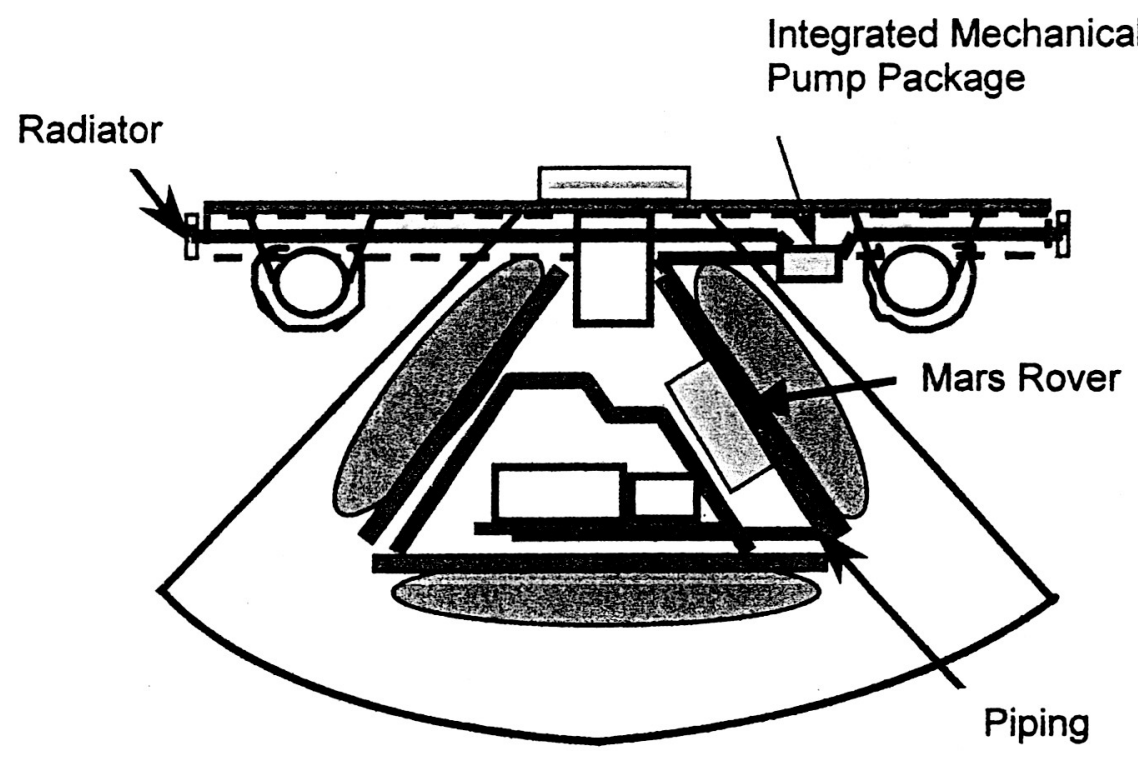

Figure 5: Mars Pathfinder Single Phase Mechanical Pumped Coolant System.

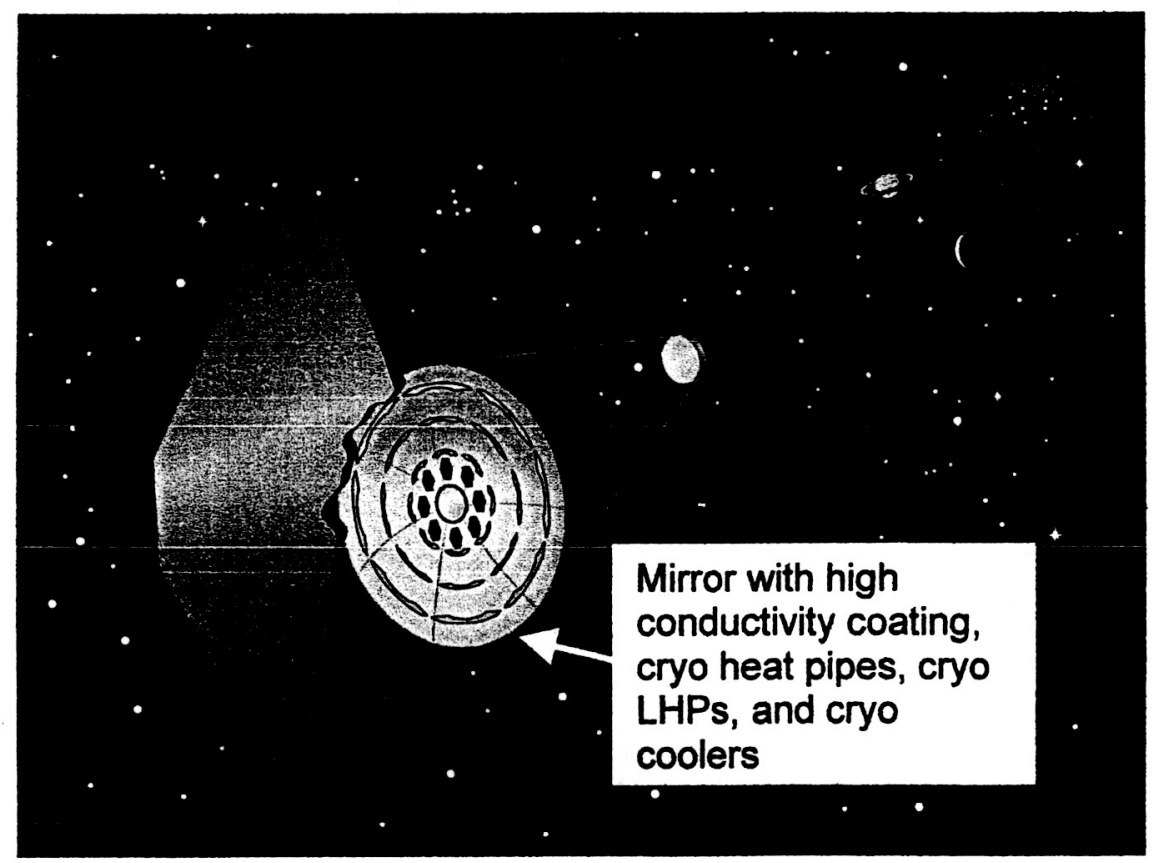

Figure 6: Integrated Cryogenic Thermal Control System for Large Telescope 


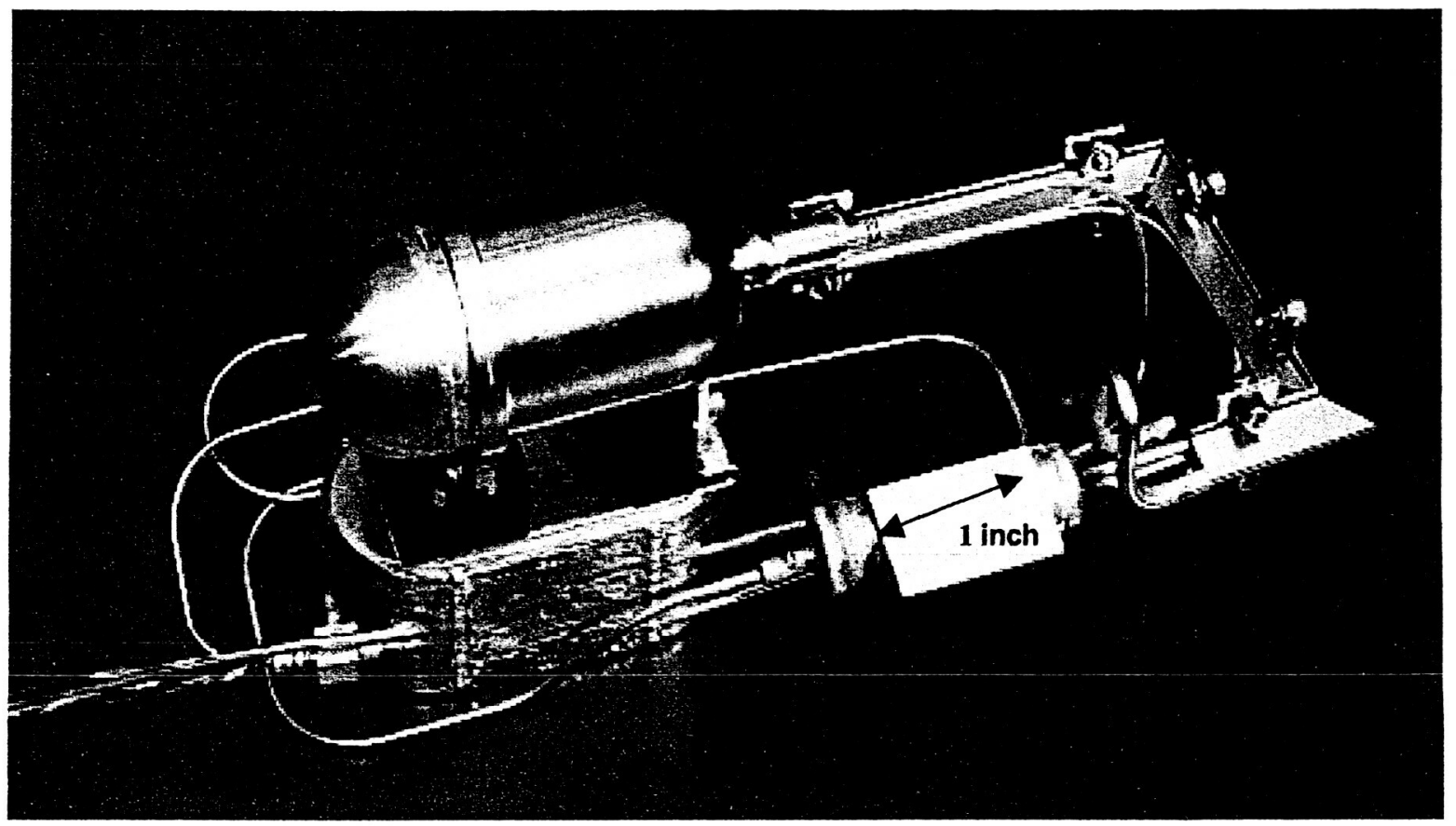

Figure 7: Nitrogen based cryogenic CPL that flew as a Shuttle experiment in 1998. 


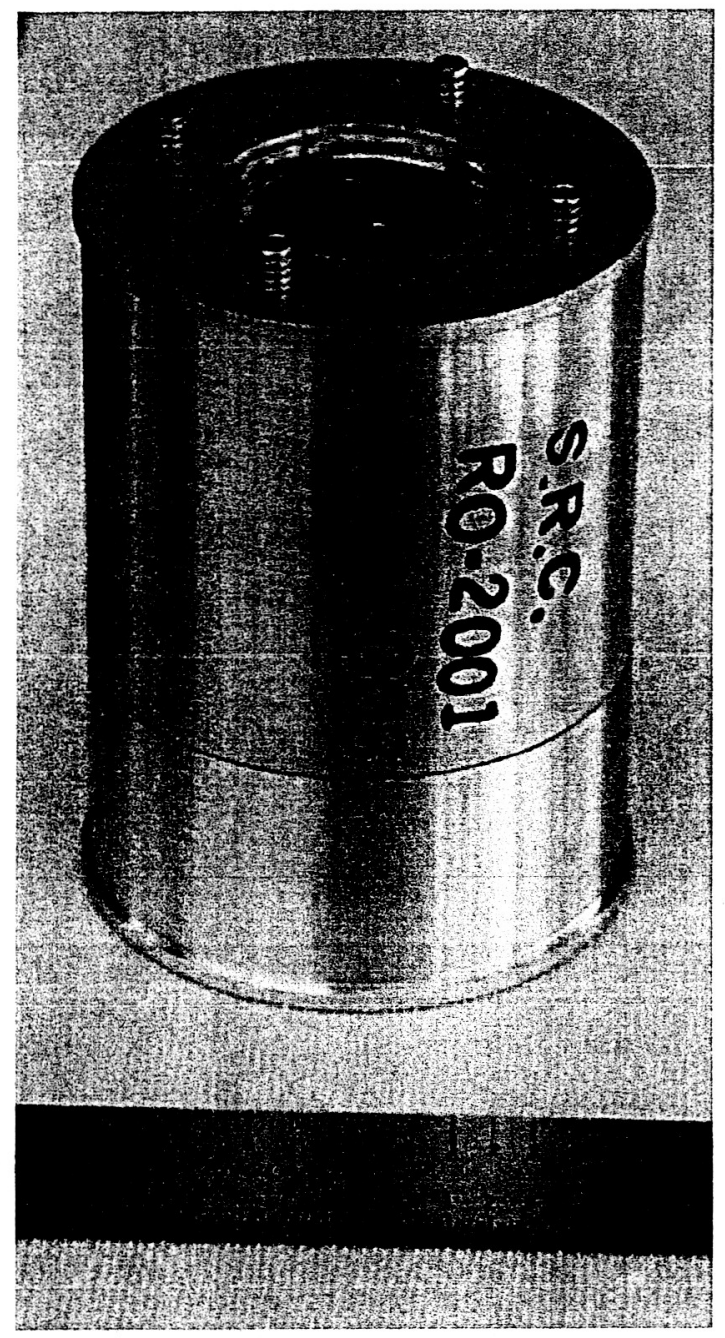

Figure 8: Wax actuated thermal switch for Mars Rover application 


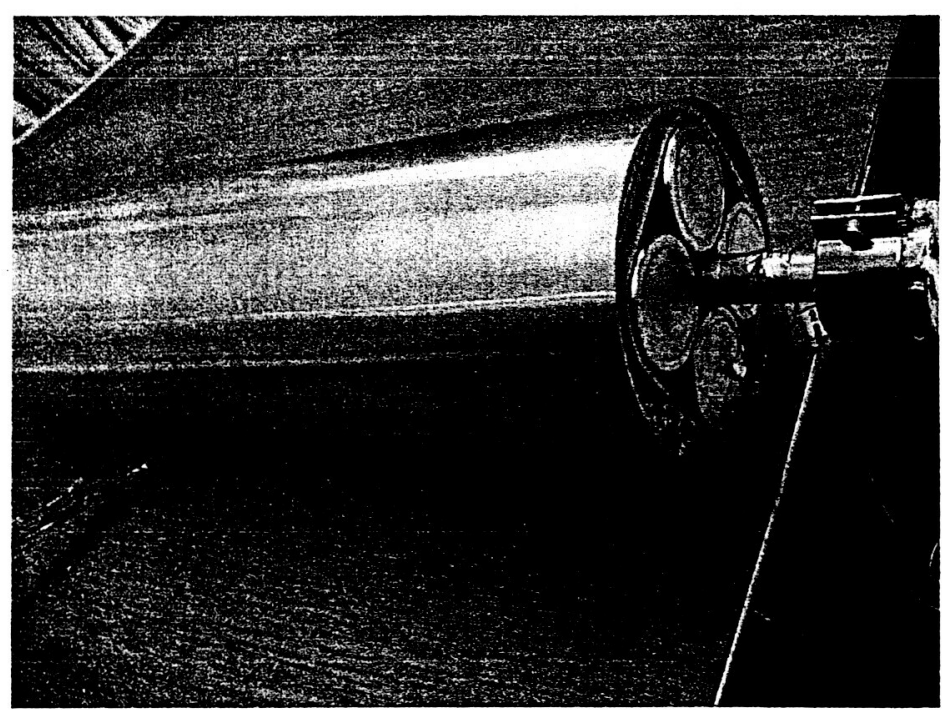

Figure 9: PCM for Mars battery application 